\title{
The role of induction and adjuvant chemotherapy in combination with concurrent chemoradiotherapy for nasopharyngeal cancer: a Bayesian network meta-analysis of published randomized controlled trials
}

This article was published in the following Dove Press journal:

OncoTargets and Therapy

5 January 2016

Number of times this article has been viewed

Hongliang Yu',*

Dayong $\mathrm{Gu}^{1, *}$

Xia $\mathrm{He}^{1}$

Xianshu $\mathrm{Gao}^{2}$

Xiuhua Bian'

'Department of Radiation Oncology, Jiangsu Cancer Hospital affiliated with Nanjing Medical University, Nanjing, ${ }^{2}$ Department of Radiation Oncology, Peking University First Hospital, Peking University, Beijing, People's Republic of China

*These authors contributed equally to this work
Correspondence: Xiuhua Bian Department of Radiation Oncology, Jiangsu Cancer Hospital affiliated with Nanjing Medical University, 42 Baiziting, Xuanwu District, Nanjing, Jiangsu 21 0009, People's Republic of China

Emailyhllovel@I63.com

\begin{abstract}
Whether the addition of induction chemotherapy (IC) or adjuvant chemotherapy (AC) to concurrent chemoradiotherapy (CCRT) is superior to CCRT alone for locally advanced nasopharyngeal cancer is unknown. A Bayesian network meta-analysis was performed to investigate the efficacy of CCRT, IC + CCRT, and CCRT + AC on locally advanced nasopharyngeal cancer. The overall survival (OS) with hazard ratios (HRs) and locoregional recurrence rates (LRRs) and distant metastasis rates (DMRs) with risk ratios (RRs) were investigated. After a comprehensive database search, eleven studies involving 2,626 assigned patients were included in this network meta-analysis. Compared with CCRT alone, IC + CCRT resulted in no significant improvement in OS or LRR and a marginal improvement in DMR (OS: HR $=0.67$, 95\% credible interval $(\mathrm{CrI}) 0.32-1.18$; LRR: $\mathrm{RR}=1.79,95 \%$ CrI 0.80-3.51; DMR: RR $=1.79$, 95\% CrI 0.24-1.04) and CCRT + AC exhibited no beneficial effects on any of the endpoints of OS, LRR, or DMR (OS: HR $=0.99,95 \%$ CrI 0.64-1.43; LRR: RR $=0.78,95 \%$ CrI 0.43-1.32; DMR: $\mathrm{RR}=0.85,95 \% \mathrm{CrI} 0.57-1.24)$. As a conclusion, for locally advanced nasopharyngeal cancer, no significant differences in the treatment efficacies of CCRT, IC + CCRT, and CCRT + $\mathrm{AC}$ were found, with the exception of a marginally significant improvement in distant control observed following IC + CCRT compared with CCRT alone.
\end{abstract}

Keywords: concurrent chemotherapy, induction chemotherapy, adjuvant chemotherapy, radiotherapy, nasopharyngeal cancer, network meta-analysis

\section{Introduction}

Nasopharyngeal carcinoma (NPC) is especially prevalent in Southeast Asian countries, southern China, and North Africa. ${ }^{1}$ Due to anatomic locations and high radiosensitivity of NPC, the mainstay treatment modality for NPC is radiotherapy (RT). For locally advanced disease, although locoregional control has been improved in recent decades by innovations in RT technology, locoregional and distant failures still impede the curing of this disease. With the aim of elevating overall survival (OS) and improving locoregional and distant control, strategies of combining chemotherapy and RT have been investigated in numerous studies. Based on the results of the pioneering clinical trial Intergroup study $0099^{2}$ and other subsequent elegant studies, ${ }^{3,4}$ platinum-based concurrent chemoradiotherapy (CCRT) was accepted as the standard treatment for locally advanced NPC. With the purpose of further improving the treatment efficacy 
and long-term prognosis, the strategy of applying additional induction chemotherapy (IC) or adjuvant chemotherapy (AC) with CCRT has been tested in several studies. ${ }^{2,5-11}$ To date however, the role of the additional IC and AC to CCRT remains controversial. The treatment effects of the addition of IC or AC to CCRT in terms of improving OS and reducing locoregional failure and distant metastasis remain unclear. Therefore, the main purpose of this study was to perform a network meta-analysis to consolidate direct and indirect evidence from published randomized controlled trials (RCTs) and explore the values of the additions of IC and AC to CCRT for the treatment of locally advanced NPC in terms of OS, locoregional recurrence rate (LRR), and distant metastasis rate (DMR).

\section{Materials and methods Data sources and research}

Relevant studies were identified via search of the PubMed database with a timeframe from inception to February 1, 2015. The studies were limited to those with human subjects that were in the English language. We used the following key text words or medical subject headings: ("nasopharyngeal" or "nasopharynx"); ("cancer" or "carcinoma" or "neoplasm"); and "radiotherapy" and ("chemotherapy" or "cisplatin" or "carboplatin" or "nedaplatin"). The reference lists of relevant reviews and meta-analyses were also manually scanned to identify potential studies for inclusion. No attempts were made to identify unpublished data.

\section{Study selection}

Studies that met the following criteria were included: 1) the enrolled patients had pathologically proven NPCs in the locally advanced disease stage without evidence of distant metastasis at presentation; 2) the patients received adequate doses of RT including a dose of at least 66 Gy to the primary lesion and involved neck; 3) studies with RCT designs with RT technologies balanced in both trial arms regardless of whether conventional RT, 3D-conformal RT, or intensive modulated radiotherapy (IMRT) was applied; 4) the patients received CCRT, IC + CCRT, or CCRT + AC in the experimental arm, and those in the control arm receiving $\mathrm{RT}$ or CCRT and the CCRT regime involved the use of platinum-based chemotherapy; and 5) the latest updated reports were included when the same patient population was reported in two or more studies.

\section{Data extraction and study quality assessment}

The identified eligible studies were independently reviewed by two authors (Hongliang Yu and Dayong Gu). From the eligible studies, we extracted the relevant data using a standardized information collecting form that included the authors' names, publication year, study country or region, comparison groups, study inclusion period, median follow-up time, number of patients enrolled, disease stage, and RT and chemotherapy regimes. The Jadad/Oxford quality scoring system was used for the quality analysis of the RCTs. ${ }^{12}$ Discrepancies in data extraction between the two reviewers were resolved by discussion.

\section{Statistical analysis}

OS was the primary endpoint of this study, and LRR and DMR were the secondary endpoints; these endpoints were defined as the time from randomization to death and the rates of locoregional and distant failure, respectively. Because the hazard ratio (HR) was the only statistical parameter that allowed for the consideration of both censoring and time to event analyses, ${ }^{13}$ HRs were used to depict the results of survival comparisons. When the HRs and 95\% confidence intervals were available from an individual study, they were extracted and directly used in our analyses. When these parameters were not available, we extracted them indirectly via the methods provided by Parma et al and Tierney et al. ${ }^{13,14}$ The risk ratios (RR) with the corresponding 95\% confidence intervals were used as result estimates in the rate comparisons. The results were directly abstracted from the included studies.

First, we performed traditional pairwise meta-analyses for the direct comparisons between the two regimens using the Review Manager software version 5.0.24 (RevMan, the Cochrane Collaboration; Oxford, UK). We calculated the pooled estimates of HRs twice using both the fixedand random-effects models. ${ }^{15}$ As described elsewhere, the random-effects model considers both the within- and between-study variations to yield more conservative results than the fixed-effects model ${ }^{15}$ and is thus more preferred when heterogeneity is available. A $P$-value $<0.05$ was considered to be statistically significant except where otherwise specified. Heterogeneity was evaluated using the $I^{2}$ test. ${ }^{16}$ When the $I^{2}$ statistic was $>50 \%$ and the $P$-value was $<0.1$, the comparison was deemed to have significant heterogeneity.

Next, network meta-analyses were performed by building a Bayesian model using the Markov chain Monte Carlo method of WinBUGS 1.4.3 (MRC Biostatistics Unit, Cambridge, UK). ${ }^{17} \mathrm{We}$ applied the calculation model proposed by Woods et a ${ }^{18}$ because this model possesses the superior feature of preserving the randomization of the RCTs and avoids potential selection bias, misleading results, and loss of available treatment comparisons. ${ }^{18}$ The treatment effects are presented as relative estimates with the corresponding $95 \%$ 
credible intervals (CrIs). ${ }^{19}$ The results of both the fixed- and random-effects models were calculated. Similar to traditional pairwise meta-analysis, the random-effects model considered both the within- and between-study variation and produced wider CrIs. Bayesian deviance information criterion statistics were used to compare the fixed and random models. This method provides a measure of model fit that penalizes model complexity; lower values are preferred, and differences of $2-5$ are considered important. ${ }^{20}$ When running the models, 50,000 iterations were applied to obtain the posterior distribution of the model parameters. The treatment ranking probabilities (ie, the best treatment, second-best treatment, etc) were also estimated according to the posterior probabilities of the results. For inconsistency evaluation, the results of the Bayesian network meta-analyses were compared with the results of the traditional pairwise meta-analyses. Nodesplitting analysis ${ }^{21}$ was applied to evaluate the inconsistencies of the closed loops in the network, and $P$-values $<0.05$ indicated significant inconsistencies.

\section{Results}

\section{Eligible studies}

During the selection process, 826 potentially relevant studies were identified through the database searches. Based on screenings of the titles and abstracts, the majority of these studies were excluded for not having RCT designs or as they were fundamental biochemical experimental research. After assessing the full texts of the 24 potentially relevant studies, 13 additional studies were excluded from the analysis. The primary reasons for these exclusions were the following: the CCRT study by Kwong et $\mathrm{al}^{22}$ did not involve a platinum-based regimen; the VUMCA I trial ${ }^{23,24}$ did not meet the criteria of containing concurrent chemotherapy in the study arms, and five additional studies were also excluded for this reason; ${ }^{25-29}$ the remaining five studies ${ }^{3,30-33}$ were excluded because more recent reports about the same populations were available. Consequently, eleven $\mathrm{RCTs}^{2,4-11,34,35}$ were finally included. A flowchart depicting the study selection process is presented in Figure 1.

\section{Study characteristics}

The characteristics of the included studies are presented in Table 1. Among the included studies, the overwhelming majority were conducted in endemic areas in East and South Asia. Only two of the studies were conducted in the US and Western European countries. ${ }^{2,8}$ The included studies were published in the years 1998 to 2013. Of the eleven RCTs, three studies ${ }^{4,34,35}$ were categorized as CCRT vs RT comparisons, five $\mathrm{e}^{2,5,7,9,11}$ were categorized as CCRT + AC vs RT comparisons, two ${ }^{6,8}$ were categorized as IC + CCRT vs CCRT comparisons, and one ${ }^{10}$ was categorized as a CCRT + $\mathrm{AC}$ vs CCRT comparison. The total number of randomly

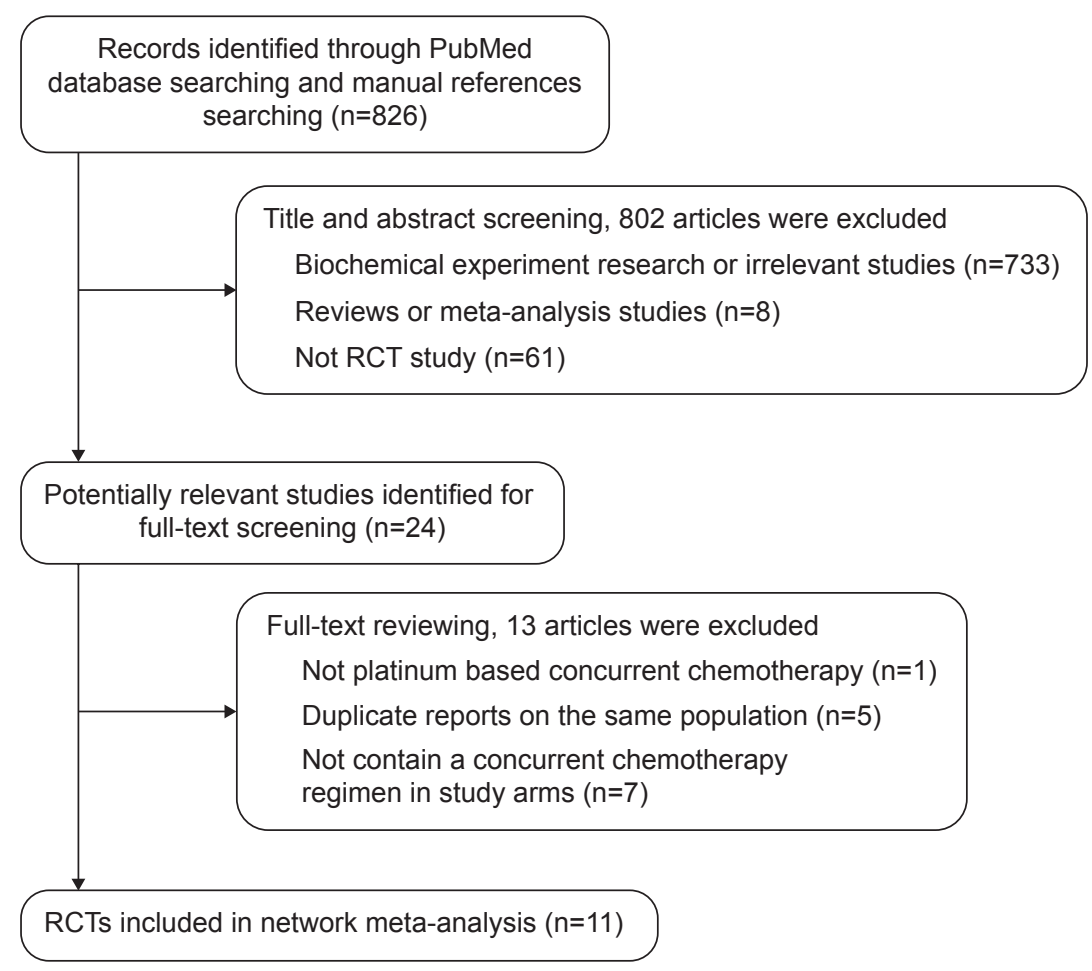

Figure I Flowchart illustrating the study selection. Abbreviation: RCT, randomized controlled trial. 


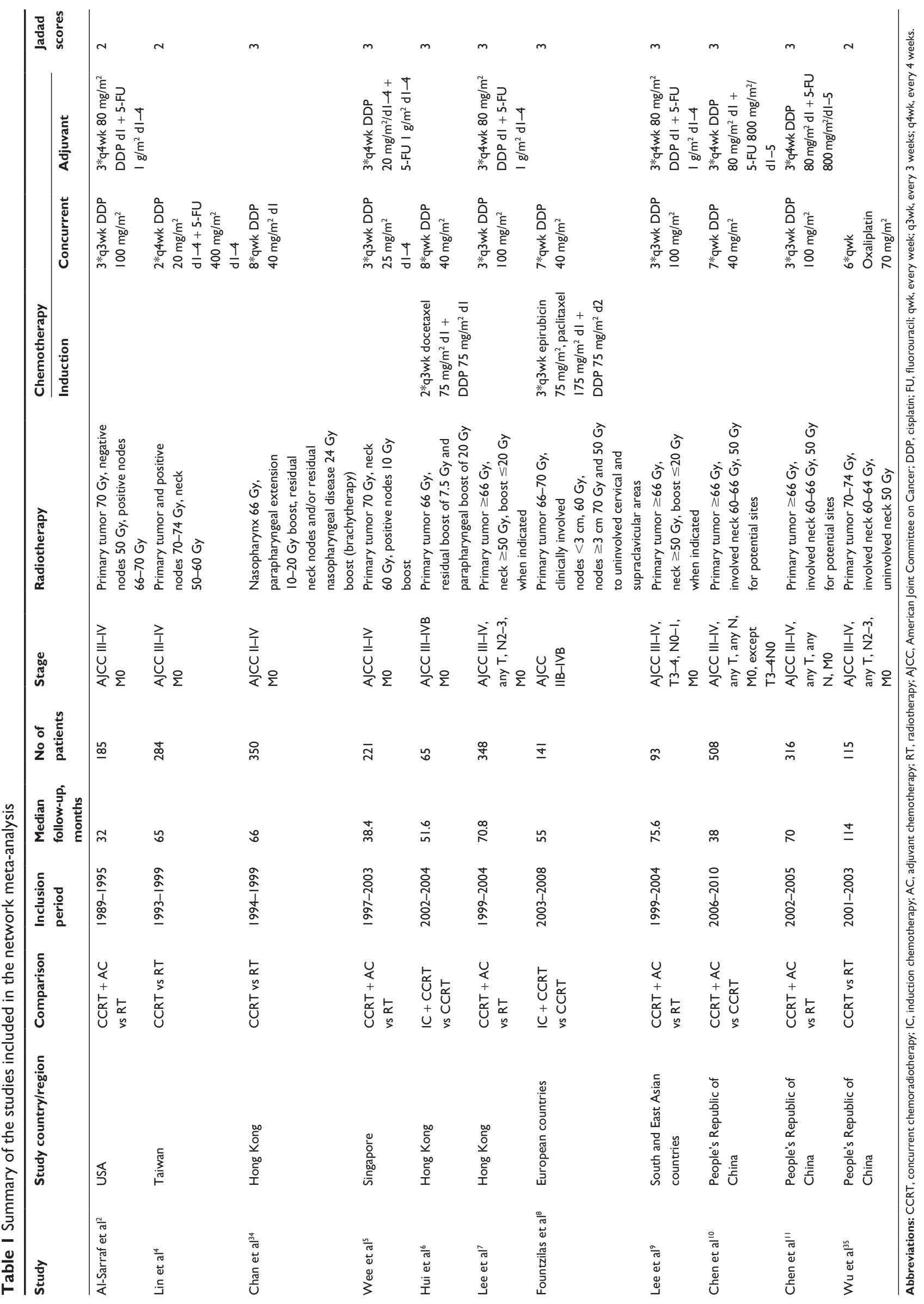


assigned patients included in this network meta-analysis was 2,626, and the disease stages ranged from II to $\mathrm{IVb}$ according to the 1997 American Joint Committee on Cancer/International Union Against Cancer staging system. Regarding RT, doses of at least 66 Gy were delivered to the primary tumors and positive nodes via conventional RT, 3D-CRT (3D conformal radiotherapy), or IMRT. According to the selection criteria, all of the induction, concurrent, and adjuvant chemotherapies in the included RCTs were platinum-based regimens.

\section{Network meta-analyses}

Figure 2 illustrates the network that was established in this study to compare the IC + CCRT, CCRT, CCRT + AC, and RT alone regimens. Figure 3 summarizes the results of the network meta-analyses. Regarding OS, Figure 3A shows that CCRT, CCRT + AC, and IC + CCRT were all significantly better than RT alone with HRs of $0.65(95 \%$ CrI: 0.45-0.91), 0.63 (95\% CrI: 0.45-0.83), and 0.43 (95\% CrI: 0.18-0.81), respectively. However, we observed no significant difference in the OSs of the CCRT, CCRT + $\mathrm{AC}$, and $\mathrm{IC}+\mathrm{CCRT}$ regimens. The distribution of ranking probabilities of each treatment regimen in terms of OS is shown in Figure 4. Based on the network results, the IC + CCRT regimen was ranked to be the best choice, CCRT + $\mathrm{AC}$ ranked second best, CCRT ranked third, and RT alone ranked last. Regarding LRR, Figure 3B showed that only CCRT + AC was observed to be significantly better than RT alone with an RR of 0.55 (95\% CrI: $0.36-0.82)$, and there were no significant differences among IC + CCRT, CCRT,

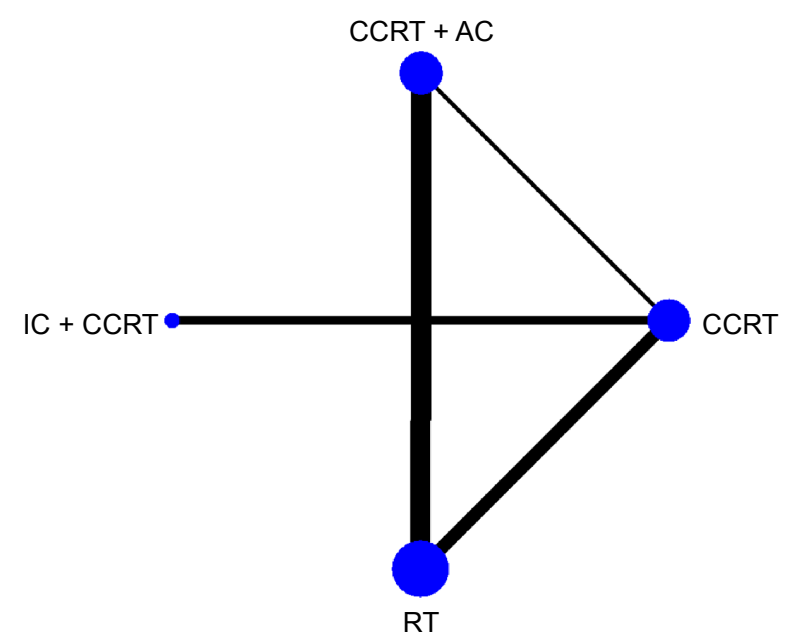

Figure 2 Network plot for multiple-treatment comparison.

Notes: The widths of the lines are proportional to the numbers of trials comparing each pair of treatments. The size of each node is proportional to the number of assigned patients (sample size).

Abbreviations: AC, adjuvantchemotherapy; CCRT, concurrentchemoradiotherapy; IC, induction chemotherapy; RT, radiotherapy. and CCRT + AC. Regarding DMR, as shown in Figure 3C, $\mathrm{IC}+\mathrm{CCRT}$ and CCRT + AC were both significantly better than RT alone with RRs of 0.42 (95\% CrI: 0.18-0.82) and 0.64 (95\% CrI: 0.48-0.83), respectively, and the beneficial effect of CCRT over RT alone was marginally significant with an RR of 0.77 (95\% CrI: 0.55-1.05). The difference in the DMRs between CCRT + AC and CCRT was not significant; however, we observed a marginally significant beneficial effect of IC + CCRT compared with CCRT alone with an RR of 0.54 (95\% CrI: $0.24,1.04)$.

The results of traditional direct comparison meta-analyses are shown in Figure 5. We confirmed a good coherence between the results of the direct and indirect comparisons, and the inconsistency analysis indicated no significant inconsistency $(P=0.67)$ between them. As shown in Figure 6, the comparison-adjusted funnel plot of the network did not exhibit any substantial asymmetry, indicating that the smallstudy effect was not significant.

\section{Discussion}

Radiation therapy is the mainstay therapeutic approach for patients with NPC. After the publication of the Intergroup 0099 study, ${ }^{2}$ which was the first RCT study to demonstrate a significant survival advantage for locally advanced NPC patients due to the addition of concurrent AC to RT, the National Comprehensive Cancer Network recommended CCRT + AC for patients with locally advanced NPC (category 2A). Several RCT studies ${ }^{4,34}$ and metaanalyses ${ }^{36,37}$ had reported beneficial effects of the addition of concurrent chemotherapy to RT. However, there are still controversies regarding the exact role of the addition of AC to CCRT due to a lack of adequate, elegantly designed head-to-head RCT studies. Very recently, Chen et $\mathrm{al}^{10}$ reported the first head-to-head Phase III RCT study that compared CCRT + AC with CCRT alone for the treatment of locally advanced NPC. The 2-year OS and failure-free survival results indicated no significant difference between CCRT + AC and CCRT alone. Based on this study, the National Comprehensive Cancer Network recommended CCRT alone as an option for locally advanced NPC (category 2B). Currently, there are major controversies regarding the effect of the addition of IC to CCRT that are reflected in the category 3 recommendation from the National Comprehensive Cancer Network. Only two Phase II RCTs by Fountzilas et $\mathrm{al}^{8}$ and Hui et $\mathrm{al}^{6}$ have investigated the efficacy of IC + CCRT compared with CCRT alone, and these studies yielded inconsistent results. Therefore, the efficacies of the additions of IC and AC to CCRT remain uncertain. 
A

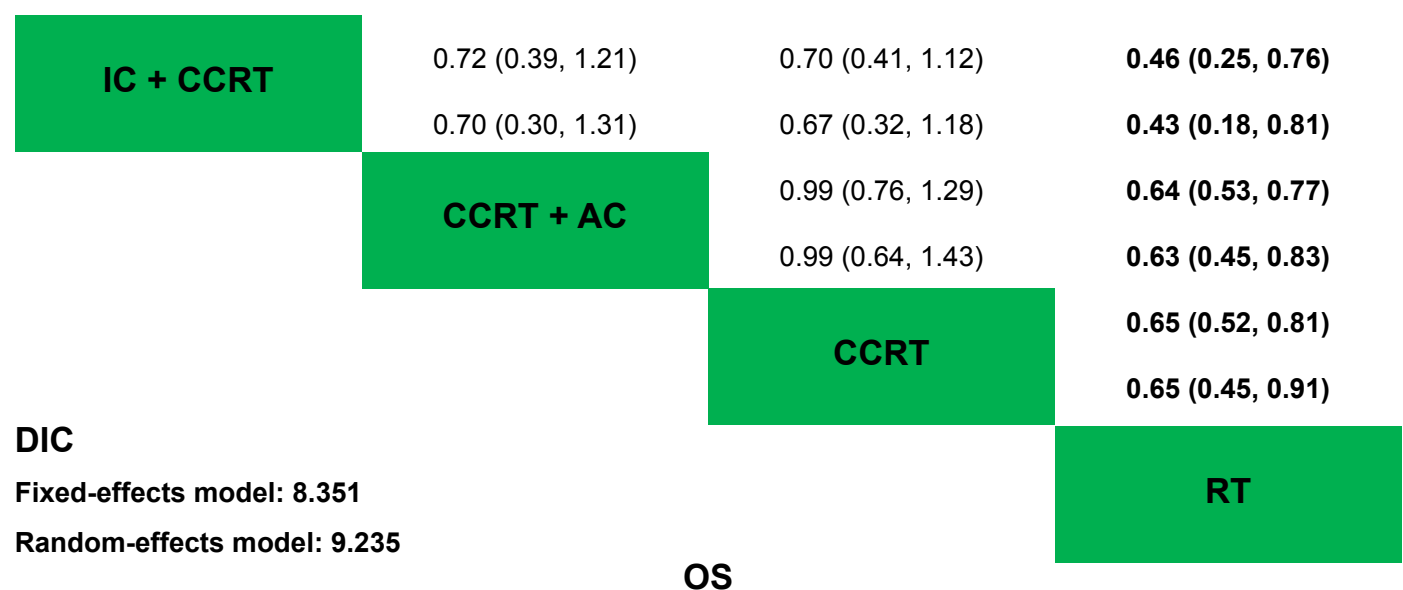

Random-effects model: 9.235

OS

B

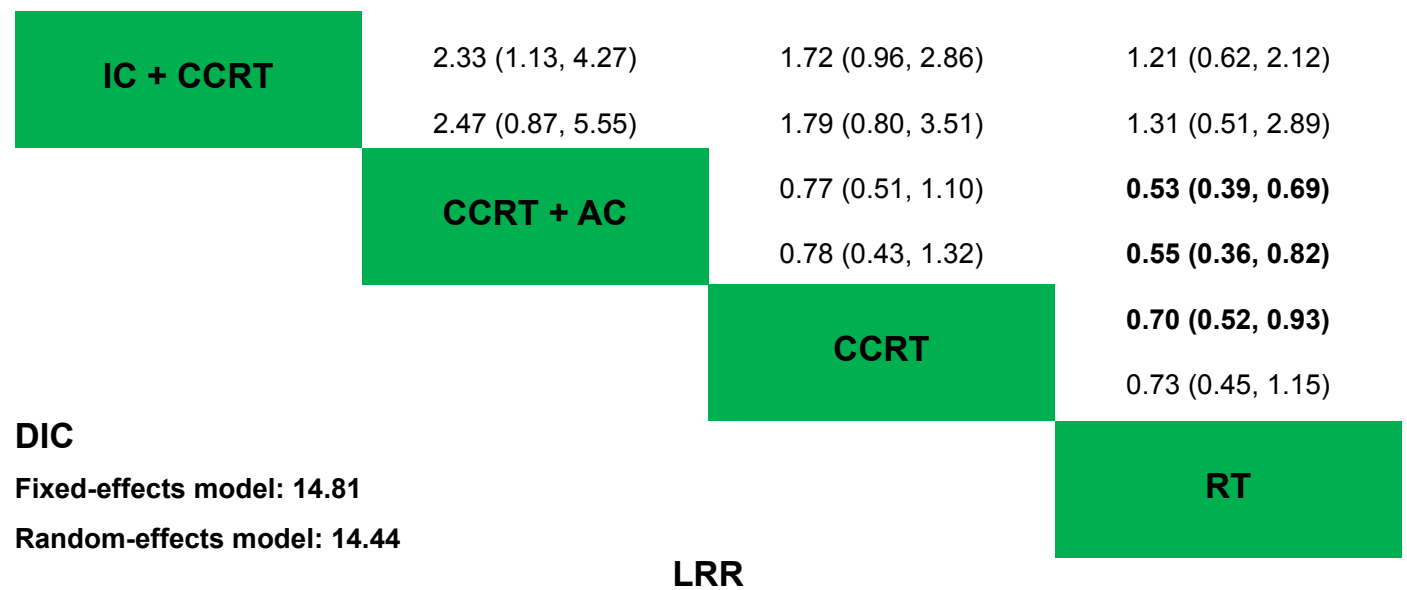

C

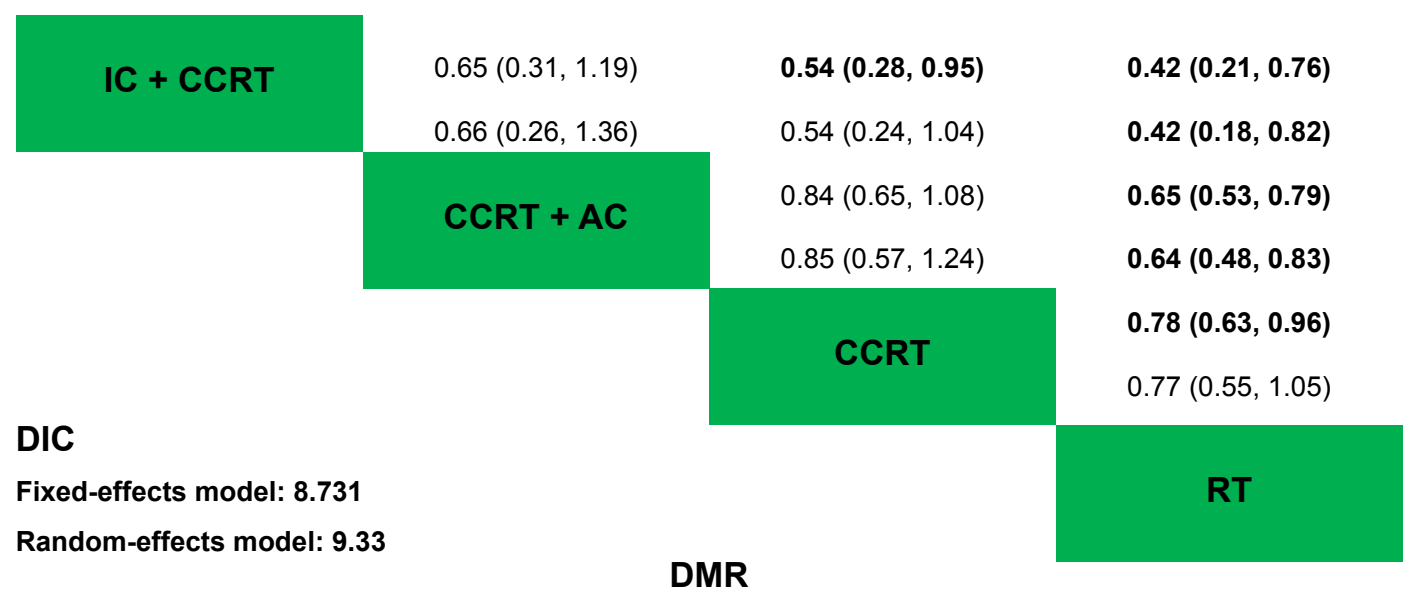

Figure 3 Results of the network meta-analyses of (A) OS, (B) LRR, and (C) DMR.

Notes: The upper triangles denote the pooled result estimates. The treatments in the rows were compared with those in the columns. In each result cell, the first and second lines contain the estimates from the fixed- and random-effects models, respectively. The numbers in parentheses indicate the corresponding $95 \%$ credible intervals. The lower triangles denote the DIC statistics from the fixed- and random-effects models. Data in bold indicates statistical significance at $P<0.05$.

Abbreviations: AC, adjuvant chemotherapy; CCRT, concurrent chemoradiotherapy; DIC, deviance information criterion; DMR, distant metastasis rate; IC, induction chemotherapy; OS, overall survival; LRR, locoregional recurrence rate; RT, radiotherapy.

New developments in network meta-analysis have provided a useful method for the collection of both direct and indirect evidence from RCTs for multitreatment comparisons. Additionally, network meta-analysis can provide probabilities for the rank positions of each treatment in a comparison. This method has substantially expanded the utility of meta-analyses for the provision of useful summaries of clinical evidence to guide guiding clinical decisions. ${ }^{38}$ To the best of our knowledge, the present Bayesian network meta-analysis is the first study to compare the efficacies of 


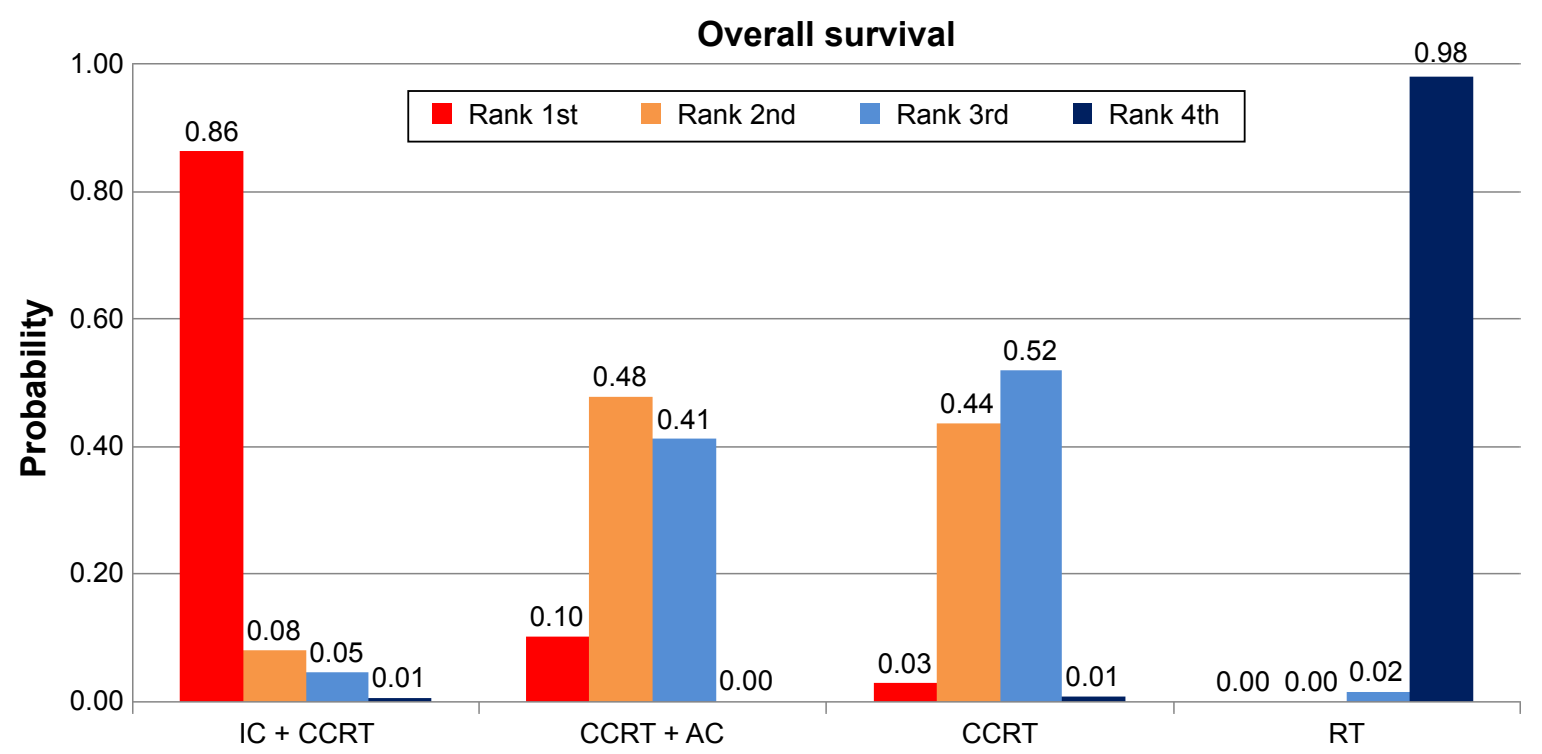

Figure 4 Rank probabilities of each treatment regimen in term of overall survival (OS) based on random-effects model.

Note: The rankings indicate the probabilities of being the best treatment choice, the second best treatment choice, and so on for the four treatment regimens in term of OS.

$\mathrm{IC}+\mathrm{CCRT}, \mathrm{CCRT}+\mathrm{AC}, \mathrm{CCRT}$, and RT alone for patients with locoregionally advanced NPC.

Based on our results, CCRT, IC + CCRT, and CCRT + $\mathrm{AC}$ all elicited significant improvements in OS compared with RT alone. CCRT exhibited a marginally significant effect on DMR but did not significantly improve LRR, while IC + CCRT had a significant effect on DMR but also failed to improve LRR. CCRT + AC significantly improved both LRR and DMR. However, no significant differences in any of the endpoints of OS, LRR, or DMR were observed among CCRT, IC + CCRT, and CCRT + AC, with the exception of the marginally significant improvement in DMR observed in the comparison of IC + CCRT and CCRT alone. Regarding the rank positions of the treatments based on OS, IC + CCRT ranked the highest, CCRT $+\mathrm{AC}$ and CCRT ranked second and third, respectively, and, as expected, RT ranked the lowest. However, it is noteworthy that in the network meta-analysis, the probabilities of the rank positions of the treatments could be produced despite the lack of statistically significant differences in the estimate results. Clinical decisions regarding treatment regimen selection should be made comprehensively and based on the individual situation of each patient.

Theoretically, IC can reduce the tumor burden, which should enhance the radiosensitivity of NPCs and might also kill subclinical micro-metastases. IC was expected to improve the OSs and reduce the LRRs and DMRs of the patients with locally advanced NPC. However, in the present study that was based on the available RCT data, we found that compared with CCRT alone, the addition of IC failed to elicit a significant improvement in LRR, and only a marginally beneficial effect on DMR was observed. Our results agree strongly with those of other studies. ${ }^{39,40}$ The possible reasons for the observed results may be that in the two included direct comparison studies, ${ }^{6,8}$ CCRT regimens and modern RT 3D-CRT/IMRT were applied. Due to the improved locoregional control achieved with CCRT and 3D-CRT/IMRT, ${ }^{39}$ the actual beneficial effect of IC on LRR was diluted and thus no significant improvement in LRR was demonstrated..$^{40}$ Furthermore, the limited numbers of patients included in the two studies was also an influential factor, and larger Phase III trials are still needed to verify the results.

In the present study, we found no significant differences between CCRT + AC and CCRT in any of the endpoints of OS, LRR, or DMR; these results are similar to those of other studies $^{40,41}$ and indicate that AC following CCRT may not remarkably improve treatment outcomes. There are two possible reasons for this result. First, the applied adjuvant cisplatin and fluorouracil regimens might have been insufficient to eradicate the micro-metastases in locally advanced NPC. Secondly, CCRT alone may be sufficient for a specific subgroup of patients with a low risk of recurrence, ${ }^{42}$ and adjuvant $\mathrm{AC}$ may not be able to elicit a further statistically significant improvement. Therefore, the application of CCRT $+\mathrm{AC}$ to all nonselected patients with locally advanced NPC may not be appropriate. In the future, new drugs and more efficient chemotherapy regimens involving AC and CCRT for the treatment of locally advanced NPC warrant further investigations. 
A

os

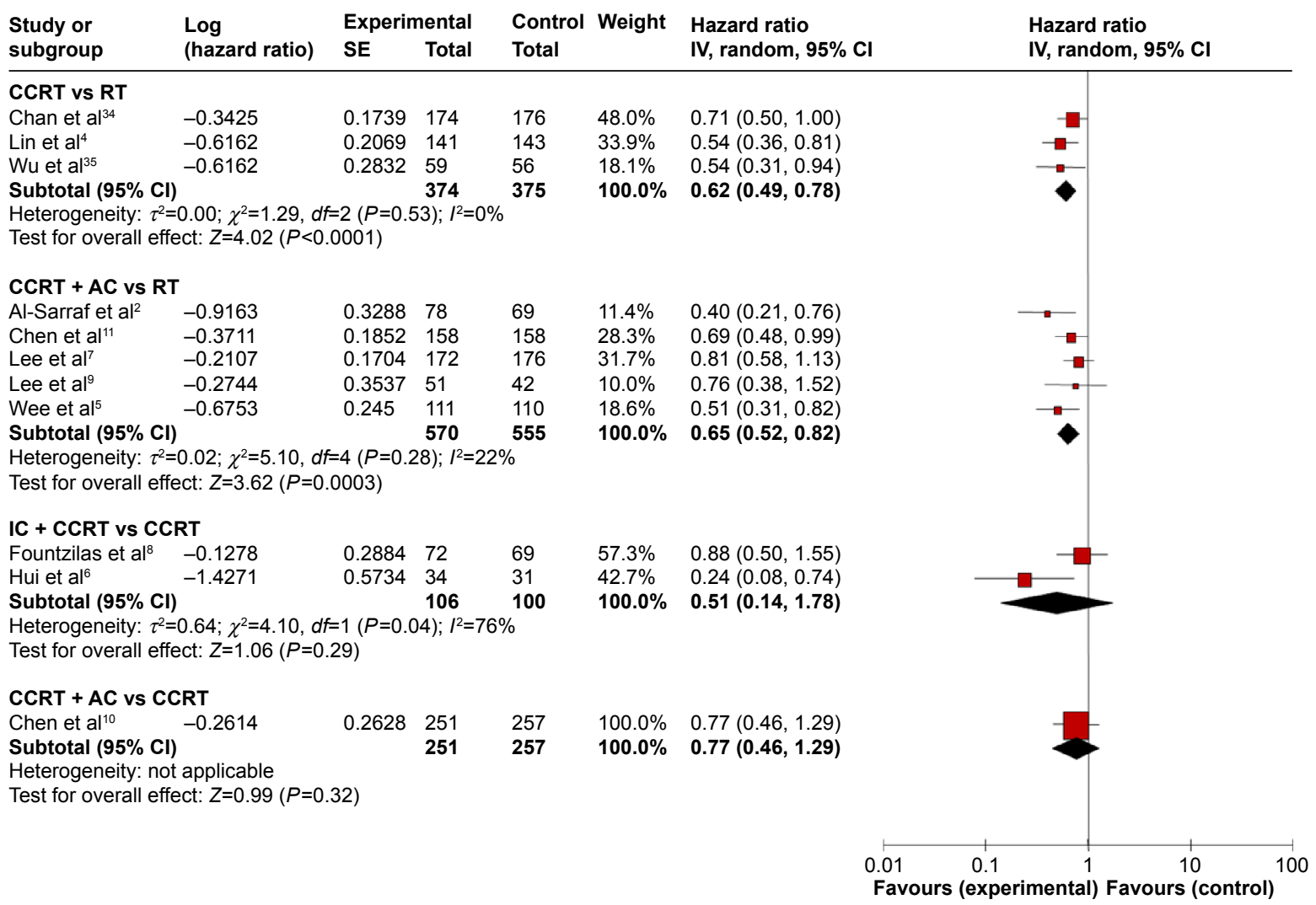

B

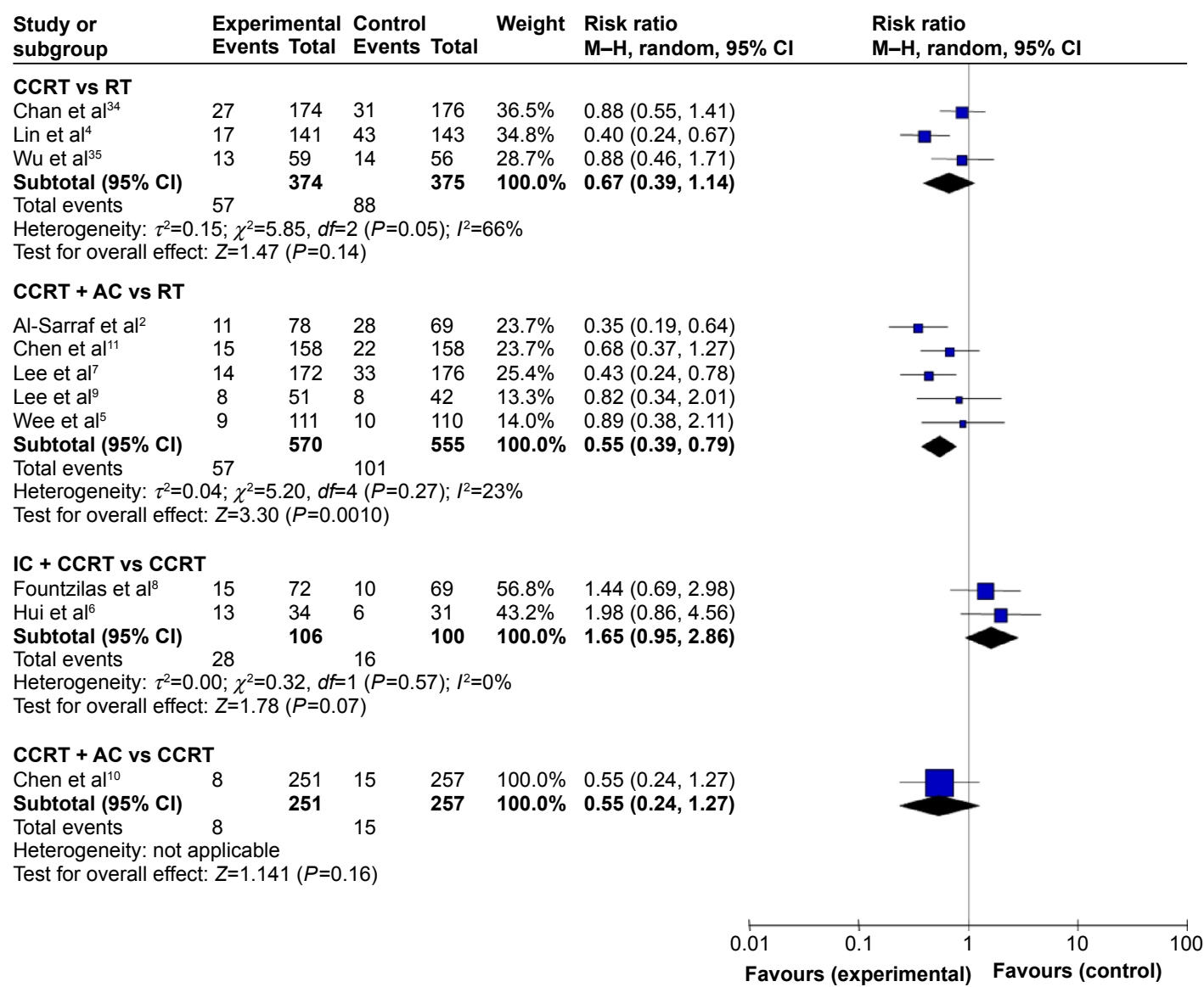

Figure 5 (Continued) 
C

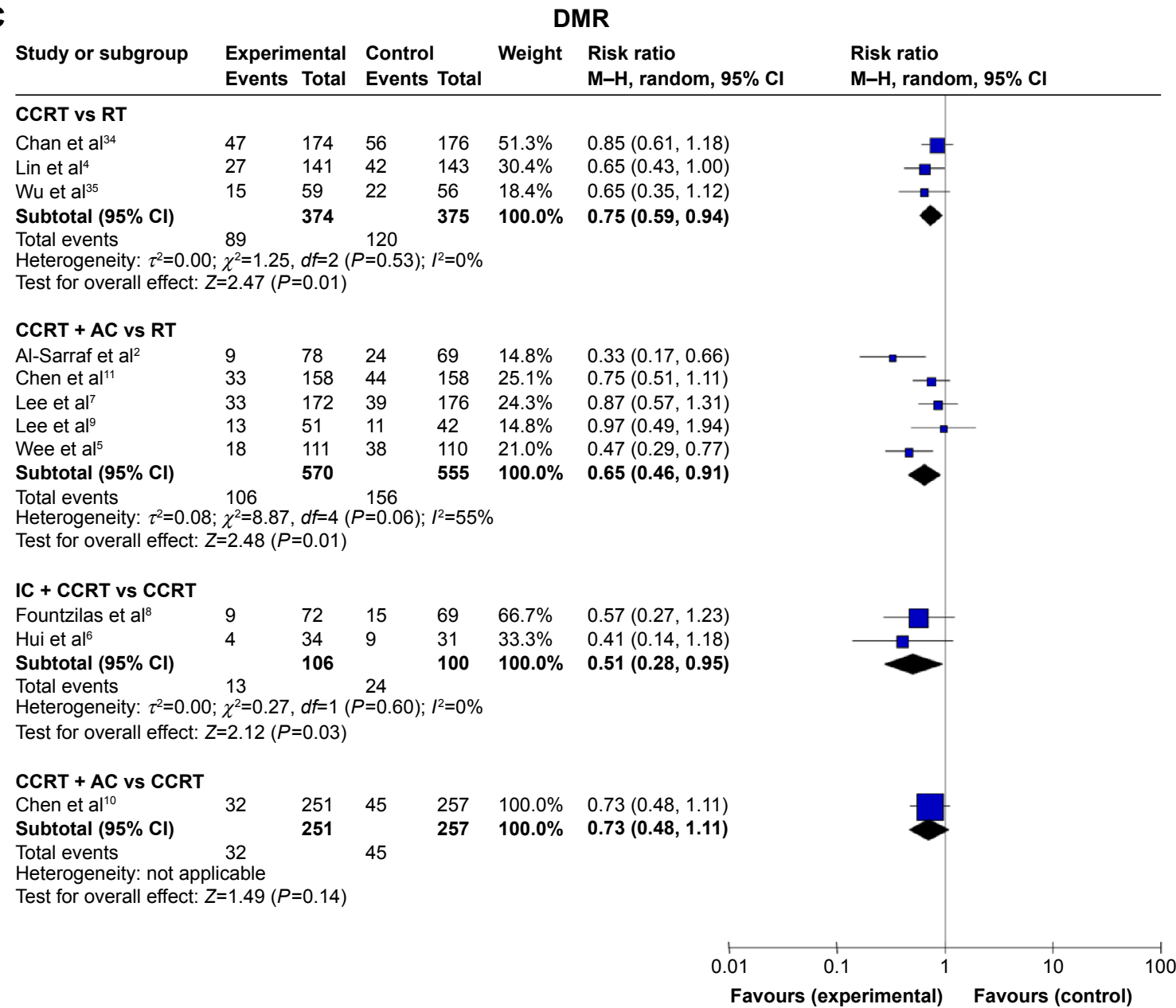

Figure 5 Forest plot of the direct comparison results.

Notes: Random-effects meta-analyses of the results of the direct comparisons of the (A) OSs, (B) LRRs, and (C) DMRs among the treatment regimens. The squares indicate the study-specific statistical weights, the horizontal lines indicate the $95 \%$ confidence intervals (Cls), and the diamonds indicate the HR or RR summary statistics with the corresponding $95 \%$ confidence intervals.

Abbreviations: AC, adjuvant chemotherapy; CCRT, concurrent chemoradiotherapy; DMR, distant metastasis rate; HR, hazard ratio; IC, induction chemotherapy; LRR, locoregional recurrence rate; OS, overall survival; RR, risk ratio; RT, radiotherapy.

Additionally, the exact efficacy of AC for the specific subgroup of patients who are at a high risk of treatment failure, that is, those with advanced node involvement (eg, N3 stage) and those with detectable EBV DNA statuses, ${ }^{42}$ remains an interesting area of study.

There are several limitations of this network meta-analysis. First, all of the data were extracted from published RCT studies, and individual patient data were not used; therefore, the quality control of the data was difficult, and analyses of the interactions between prognostic factors and treatment effects were not possible. Second, due to the lack of available appropriate head-to-head RCTs, the quantity of studies included for one specific comparison was small; especially, there was only one study included in the subgroup comparing CCRT + AC vs CCRT, even though we have done a thorough examination of the database. More studies focusing on this topic are warranted to consolidate the conclusion. Third, the qualities of the included RCTs varied. However, our comparison-adjusted funnel plot did not exhibit any asymmetry, indicating that there was no significant publication bias; this result was mainly due to the small-study effect, which tends to lead to greater treatment effects compared with larger studies. ${ }^{43,44}$ Finally, the technologies of the radiotherapies that were applied in the included studies evolved from conventional RT to 3D-CRT or IMRT. The utilized technologies even varied within the timeframes of some of the individual RCTs. ${ }^{7}$ Such variations may have notably affected the local control, OS, and even the scale of the treatment effects of chemotherapy. However, to date, there are very few studies that have focused on this issue.

In conclusion, our Bayesian network meta-analysis confirmed the significant beneficial effects of CCRT, IC + CCRT, and CCRT + AC compared with RT alone in terms of the OS of patients with locally advanced NPC. There were 


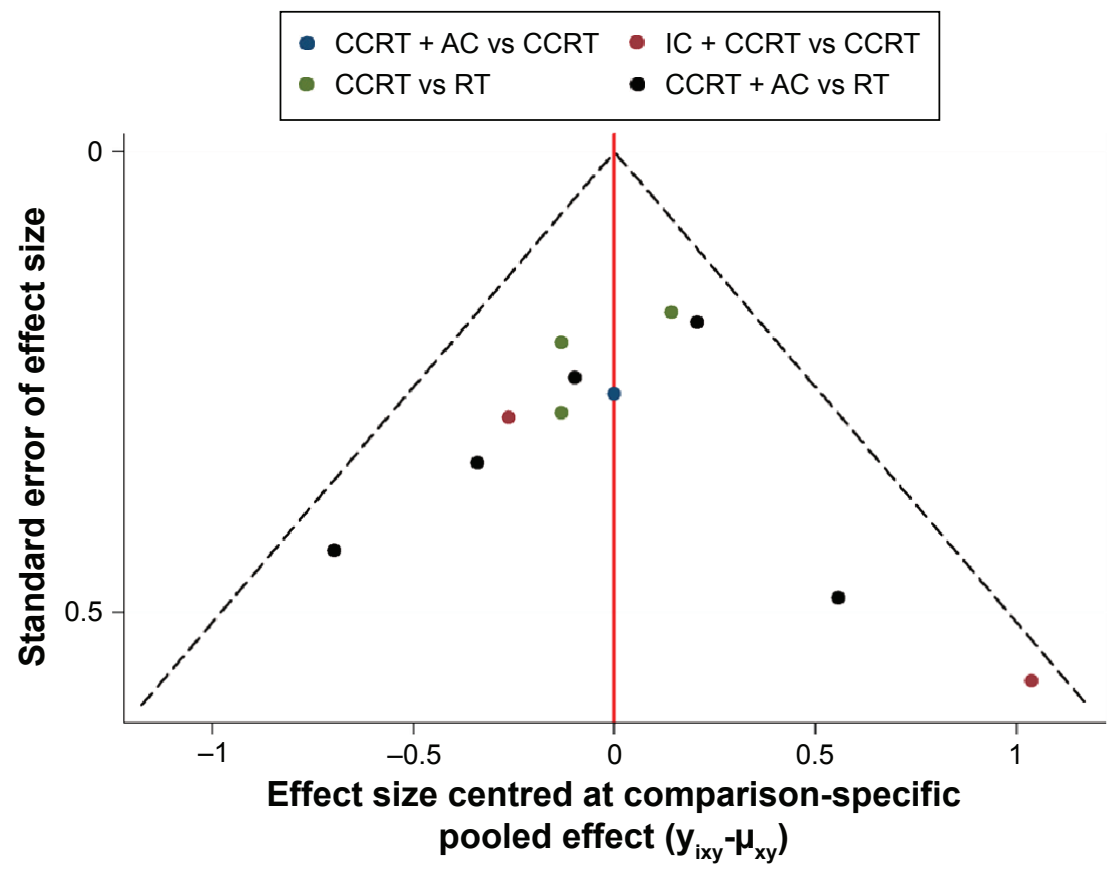

Figure 6 Comparison-adjusted funnel plot for the network meta-analysis.

Notes: The different colors correspond to different comparisons. The lack of substantial asymmetry indicates that the small-study effect was not significant. Abbreviations: AC, adjuvant chemotherapy; CCRT, concurrent chemoradiotherapy; IC, induction chemotherapy; RT, radiotherapy.

no significant differences among CCRT, IC + CCRT, and CCRT + AC in any of the endpoints of OS, LRR, or DMR, with the exception of a marginally significant improvement in DMR with IC + CCRT compared to CCRT alone. The exact roles of the additions of IC and AC to CCRT for the treatment of locally advanced NPC warrant further investigation.

\section{Acknowledgments}

We thank Shuping Wei for the critical reading of the manuscript and English language assistance. This work was supported by the Jiangsu Provincial Natural Science Fund (No BK20141018), the “333” Project of Jiangsu Province (No BRA2013292), the Six Major Talent Peak Project of Jiangsu Province (class B, No WS-047), and the Natural Science Foundation of China (Nos 30670619, 81072017).

\section{Disclosure}

The authors report no conflicts of interest in this work.

\section{References}

1. Wei WI, Sham JS. Nasopharyngeal carcinoma. Lancet. 2005;365(9476): 2041-2054.

2. Al-Sarraf M, LeBlanc M, Giri PG, et al. Chemoradiotherapy versus radiotherapy in patients with advanced nasopharyngeal cancer: phase III randomized Intergroup study 0099. J Clin Oncol. 1998;16(4):1310-1317.

3. Chan AT, Teo PM, Ngan RK, et al. Concurrent chemotherapyradiotherapy compared with radiotherapy alone in locoregionally advanced nasopharyngeal carcinoma: progression-free survival analysis of a phase III randomized trial. J Clin Oncol. 2002;20(8): $2038-2044$.
4. Lin JC, Jan JS, Hsu CY, Liang WM, Jiang RS, Wang WY. Phase III study of concurrent chemoradiotherapy versus radiotherapy alone for advanced nasopharyngeal carcinoma: positive effect on overall and progression-free survival. J Clin Oncol. 2003;21(4):631-637.

5. Wee J, Tan EH, Tai BC, et al. Randomized trial of radiotherapy versus concurrent chemoradiotherapy followed by adjuvant chemotherapy in patients with American Joint Committee on Cancer/International Union against cancer stage III and IV nasopharyngeal cancer of the endemic variety. J Clin Oncol. 2005;23(27):6730-6738.

6. Hui EP, Ma BB, Leung SF, et al. Randomized phase II trial of concurrent cisplatin-radiotherapy with or without neoadjuvant docetaxel and cisplatin in advanced nasopharyngeal carcinoma. J Clin Oncol. 2009;27(2):242-249.

7. Lee AW, Tung SY, Chua DT, et al. Randomized trial of radiotherapy plus concurrent-adjuvant chemotherapy vs radiotherapy alone for regionally advanced nasopharyngeal carcinoma. J Natl Cancer Inst. 2010;102(15):1188-1198.

8. Fountzilas G, Ciuleanu E, Bobos M, et al. Induction chemotherapy followed by concomitant radiotherapy and weekly cisplatin versus the same concomitant chemoradiotherapy in patients with nasopharyngeal carcinoma: a randomized phase II study conducted by the Hellenic Cooperative Oncology Group (HeCOG) with biomarker evaluation. Ann Oncol. 2012;23(2):427-435.

9. Lee AW, Tung SY, Chan AT, et al. A randomized trial on addition of concurrent-adjuvant chemotherapy and/or accelerated fractionation for locally-advanced nasopharyngeal carcinoma. Radiother Oncol. 2011;98(1):15-22.

10. Chen L, Hu CS, Chen XZ, et al. Concurrent chemoradiotherapy plus adjuvant chemotherapy versus concurrent chemoradiotherapy alone in patients with locoregionally advanced nasopharyngeal carcinoma: a phase 3 multicentre randomised controlled trial. Lancet Oncol. 2012;13(2):163-171.

11. Chen Y, Sun Y, Liang SB, et al. Progress report of a randomized trial comparing long-term survival and late toxicity of concurrent chemoradiotherapy with adjuvant chemotherapy versus radiotherapy alone in patients with stage III to IVB nasopharyngeal carcinoma from endemic regions of China. Cancer. 2013;119(12):2230-2238. 
12. Jadad AR, Moore RA, Carroll D, et al. Assessing the quality of reports of randomized clinical trials: is blinding necessary? Control Clin Trials. 1996;17(1):1-12.

13. Parmar MK, Torri V, Stewart L. Extracting summary statistics to perform meta-analyses of the published literature for survival endpoints. Stat Med. 1998;17(24):2815-2834.

14. Tierney JF, Stewart LA, Ghersi D, Burdett S, Sydes MR. Practical methods for incorporating summary time-to-event data into metaanalysis. Trials. 2007;8:16.

15. DerSimonian R, Laird N. Meta-analysis in clinical trials. Control Clin Trials. 1986;7(3):177-188.

16. Higgins JP, Thompson SG, Deeks JJ, Altman DG. Measuring inconsistency in meta-analyses. BMJ. 2003;327(7414):557-560.

17. Fryback DG, Stout NK, Rosenberg MA. An elementary introduction to Bayesian computing using WinBUGS. Int J Technol Assess Health Care. 2001;17(1):98-113.

18. Woods BS, Hawkins N, Scott DA. Network meta-analysis on the loghazard scale, combining count and hazard ratio statistics accounting for multi-arm trials: a tutorial. BMC Med Res Methodol. 2010;10:54.

19. Wandel S, Juni P, Tendal B, et al. Effects of glucosamine, chondroitin, or placebo in patients with osteoarthritis of hip or knee: network metaanalysis. BMJ. 2010;341:c4675.

20. Spiegelhalter DJ, Best NG, Carlin BP, Van Der Linde A. Bayesian measures of model complexity and fit. J R Statist Soc B. 2002;64(4): 583-639.

21. Higgins J, Jackson D, Barrett J, Lu G, Ades A, White I. Consistency and inconsistency in network meta-analysis: concepts and models for multi-arm studies. Res Synth Methods. 2012;3(2):98-110.

22. Kwong DL, Sham JS, Au GK, et al. Concurrent and adjuvant chemotherapy for nasopharyngeal carcinoma: a factorial study. J Clin Oncol. 2004;22(13):2643-2653.

23. International Nasopharynx Cancer Study G, Trial VI. Preliminary results of a randomized trial comparing neoadjuvant chemotherapy (cisplatin, epirubicin, bleomycin) plus radiotherapy vs radiotherapy alone in stage IV $(>$ or $=\mathrm{N} 2, \mathrm{M} 0)$ undifferentiated nasopharyngeal carcinoma: a positive effect on progression-free survival. Int J Radiat Oncol Biol Phys. 1996;35(3):463-469.

24. Be G. Final results of the VUMCA I randomized trial comparing neo adjuvant chemotherapy (CT) (BEC) plus radiotherapy (RT) to RT alone in undifferentiated nasopharyngeal carcinoma (UCNT) (Meeting abstract). Paper presented at: Proc Annu Meet Am Soc Clin Oncol. 1998. Volume 17. A1482.

25. Chan AT, Teo PM, Leung TW, et al. A prospective randomized study of chemotherapy adjunctive to definitive radiotherapy in advanced nasopharyngeal carcinoma. Int J Radiat Oncol Biol Phys. 1995;33(3): 569-577.

26. Chi KH, Chang YC, Guo WY, et al. A phase III study of adjuvant chemotherapy in advanced nasopharyngeal carcinoma patients. Int J Radiat Oncol Biol Phys. 2002;52(5):1238-1244.

27. Chua DT, Sham JS, Choy D, et al. Preliminary report of the AsianOceanian Clinical Oncology Association randomized trial comparing cisplatin and epirubicin followed by radiotherapy versus radiotherapy alone in the treatment of patients with locoregionally advanced nasopharyngeal carcinoma. Asian-Oceanian Clinical Oncology Association Nasopharynx Cancer Study Group. Cancer. 1998;83(11):2270-2283.

28. Hareyama M, Sakata K, Shirato H, et al. A prospective, randomized trial comparing neoadjuvant chemotherapy with radiotherapy alone in patients with advanced nasopharyngeal carcinoma. Cancer. 2002; 94(8):2217-2223.

29. Ma J, Mai HQ, Hong MH, et al. Results of a prospective randomized trial comparing neoadjuvant chemotherapy plus radiotherapy with radiotherapy alone in patients with locoregionally advanced nasopharyngeal carcinoma. J Clin Oncol. 2001;19(5):1350-1357.
30. Lee AW, Lau WH, Tung SY, et al. Preliminary results of a randomized study on therapeutic gain by concurrent chemotherapy for regionally-advanced nasopharyngeal carcinoma: NPC-9901 Trial by the Hong Kong Nasopharyngeal Cancer Study Group. J Clin Oncol. 2005;23(28):6966-6975.

31. Lee AW, Tung SY, Chan AT, et al. Preliminary results of a randomized study (NPC-9902 Trial) on therapeutic gain by concurrent chemotherapy and/or accelerated fractionation for locally advanced nasopharyngeal carcinoma. Int J Radiat Oncol Biol Phys. 2006;66(1):142-151.

32. Chen Y, Liu MZ, Liang SB, et al. Preliminary results of a prospective randomized trial comparing concurrent chemoradiotherapy plus adjuvant chemotherapy with radiotherapy alone in patients with locoregionally advanced nasopharyngeal carcinoma in endemic regions of China. Int J Radiat Oncol Biol Phys. 2008;71(5):1356-1364.

33. Zhang L, Zhao C, Peng PJ, et al. Phase III study comparing standard radiotherapy with or without weekly oxaliplatin in treatment of locoregionally advanced nasopharyngeal carcinoma: preliminary results. J Clin Oncol. 2005;23(33):8461-8468.

34. Chan AT, Leung SF, Ngan RK, et al. Overall survival after concurrent cisplatin-radiotherapy compared with radiotherapy alone in locoregionally advanced nasopharyngeal carcinoma. J Natl Cancer Inst. 2005; 97(7):536-539.

35. Wu X, Huang P, Peng P, et al. Long-term follow-up of a phase III study comparing radiotherapy with or without weekly oxaliplatin for locoregionally advanced nasopharyngeal carcinoma. Ann Oncol. 2013; 24(8):2131-2136.

36. Baujat B, Audry H, Bourhis J, et al. Chemotherapy in locally advanced nasopharyngeal carcinoma: an individual patient data meta-analysis of eight randomized trials and 1,753 patients. Int J Radiat Oncol Biol Phys. 2006;64(1):47-56.

37. Zhang L, Zhao C, Ghimire B, et al. The role of concurrent chemoradiotherapy in the treatment of locoregionally advanced nasopharyngeal carcinoma among endemic population: a meta-analysis of the phase III randomized trials. BMC Cancer. 2010;10:558.

38. Ades AE, Sculpher M, Sutton A, et al. Bayesian methods for evidence synthesis in cost-effectiveness analysis. Pharmacoeconomics. 2006; 24(1):1-19.

39. Lee N, Xia P, Quivey JM, et al. Intensity-modulated radiotherapy in the treatment of nasopharyngeal carcinoma: an update of the UCSF experience. Int J Radiat Oncol Biol Phys. 2002;53(1):12-22.

40. OuYang PY, Xie C, Mao YP, et al. Significant efficacies of neoadjuvant and adjuvant chemotherapy for nasopharyngeal carcinoma by metaanalysis of published literature-based randomized, controlled trials. Ann Oncol. 2013;24(8):2136-2146.

41. Chen YP, Wang ZX, Chen L, et al. A Bayesian network meta-analysis comparing concurrent chemoradiotherapy followed by adjuvant chemotherapy, concurrent chemoradiotherapy alone and radiotherapy alone in patients with locoregionally advanced nasopharyngeal carcinoma. Ann Oncol. 2015;26(1):205-211.

42. Lin JC, Liang WM, Jan JS, Jiang RS, Lin AC. Another way to estimate outcome of advanced nasopharyngeal carcinoma - is concurrent chemoradiotherapy adequate? Int J Radiat Oncol Biol Phys. 2004;60(1):156-164.

43. Egger M, Davey Smith G, Schneider M, Minder C. Bias in meta-analysis detected by a simple, graphical test. BMJ. 1997;315(7109):629-634.

44. Nuesch E, Trelle S, Reichenbach S, et al. Small study effects in metaanalyses of osteoarthritis trials: meta-epidemiological study. BMJ. 2010;341:c3515. 


\section{Publish your work in this journal}

OncoTargets and Therapy is an international, peer-reviewed, open access journal focusing on the pathological basis of all cancers, potential targets for therapy and treatment protocols employed to improve the management of cancer patients. The journal also focuses on the impact of management programs and new therapeutic agents and protocols on

patient perspectives such as quality of life, adherence and satisfaction. The manuscript management system is completely online and includes a very quick and fair peer-review system, which is all easy to use. Visit http://www.dovepress.com/testimonials.php to read real quotes from published authors.

Submit your manuscript here: http://www.dovepress.com/oncotargets-and-therapy-journal 